\title{
Fin d'empire, apocalypse et résurrectiondans le roman de Mikhaïl Chichkine, Le Cheveu de Vénus
}

\section{Isabelle Després}

\section{OpenEdition}

\section{Journals}

Édition électronique

URL : http://journals.openedition.org/recherchestravaux/527

DOI : 10.4000/recherchestravaux.527

ISSN : 1969-6434

Éditeur

UGA Éditions/Université Grenoble Alpes

\section{Édition imprimée}

Date de publication : 15 mai 2012

Pagination : 173-186

ISBN : 978-2-84310-227-1

ISSN : 0151-1874

Référence électronique

Isabelle Després, "Fin d'empire, apocalypse et résurrectiondans le roman de Mikhaïl Chichkine, Le Cheveu de Vénus », Recherches \& Travaux [En ligne], 80 | 2012, mis en ligne le 15 novembre 2013,

consulté le 08 septembre 2020. URL : http://journals.openedition.org/recherchestravaux/527 ; DOI https://doi.org/10.4000/recherchestravaux.527 
Isabelle DesPRÉS

Université de Grenoble

\section{Fin d'empire, apocalypse et résurrection dans le roman de Mikhail Chichkine, Le Cheveu de Vénus}

Le Cheveu de Vénus ${ }^{\mathrm{I}}$ est le troisième roman de Mikhaïl Chichkine, écrivain russe contemporain, né en 196I à Moscou, ayant émigré à Zurich à la fin des années 1990. Proche par le style du roman précédent, La Prise d'Izmail ${ }^{2}$, ce "roman polyphonique d'une complexité raffinée ${ }^{3}$ " a un caractère largement autobiographique, puisque le héros narrateur, qui se surnomme lui-même le "drogman ${ }^{4}$ », est précisément un émigré russe, travaillant comme interprète pour la police suisse, et en particulier pour le chef d'un service chargé d'examiner les récits, parfois affabulateurs, des demandeurs d'asile en provenance de l'ex-URSS.

L'ensemble de ces récits compose un tableau à la fois réaliste et apocalyptique de l'ex-empire soviétique, en pleine déliquescence. En outre, derrière la réalité post-soviétique, un autre tableau se dessine, celui de la période soviétique, qui n'est pas moins décadente que l'époque contemporaine. Enfin, un

I. M. Chichkine, Венерин волос, Вагриус, 2006; (en français : Le Cheveu de Vénus, trad. du russe par L. Troubetzkoy, Fayard, 2007). Toutes les références seront données dans cette édition.

2. La Prise d'Izmail avait obtenu le Booker Prize russe en 1999. Cependant, en Russie, les romans de Chichkine suscitent la polémique; de nombreux critiques littéraires "patriotes" sont hostiles à Chichkine, lui reprochant son style «imprécis» et influencé par l'Occident.

3. Voir la note annonçant sa parution en italien sur le site suisse <www.culturactif.ch/ livredumois/marso7shishkin.htm>. Voir aussi B. Yari, «Le cheveu de Vénus», compte rendu traduit et adapté par Fr. Biamonte sur le site <http://www.culturactif.ch/livredumois/septo7chichkine.htm)>.

4. Le mot russe est un archaïsme signifiant «l'interprète", "celui qui fait le lien", "qui explique et clarifie». 
autre empire, celui des tsars, se dévoile dans tout le tragique de sa chute, grâce au procédé du "roman dans le roman" que constitue le Journal de Bella et l'histoire de sa vie, lue, et plus ou moins romancée, par le drogman lui-même. Deux écroulements d'empires se superposent, ce qui a pour conséquence de figer le temps, d'arrêter l'apocalypse, de permettre un retour sur image.

Par la force de la conscience, de la mémoire et du souvenir, le temps du roman s'inverses. La vieille femme décatie retrouve une forme de vie grâce à la lecture de son journal par le héros, qui de son côté parcourt rétrospectivement l'itinéraire de sa propre vie, en revenant à Rome, la ville impériale, ici ville de l'amour, à laquelle aboutissent plusieurs des lignes du roman. Dans la conscience du héros, à travers ses lectures et ses souvenirs, se produit ainsi une remontée historique, qui le mène, avec son lecteur, jusqu'à l'Antiquité grecque, l'Empire perse et Babylone.

\section{La décadence et l'Apocalypse}

Les histoires que les réfugiés russes racontent pour obtenir le droit de vivre en Suisse sont toutes plus atroces les unes que les autres. La violence et les injustices sont omniprésentes. La précision des détails ne laisse pourtant aucun doute sur le réalisme des situations décrites ${ }^{6}$. Il s'agit bien de la chronique des événements ordinaires dans la Russie des années I990, livrée à elle-même après l'effondrement de l'empire, désemparée, victime de la dissolution des mœurs. La violence s'exprime de façon directe et crue. Il s'agit d'abord du viol d'un jeune garçon, dans un orphelinat, commis par le directeur luimême. Plus loin, c'est le viol de jeunes femmes insouciantes, après une partie de brochettes trop arrosée avec des collègues. Ailleurs on trouve des histoires cruelles de bizutage de gamins en camp de redressement (p. 49). L'absence de droits démocratiques, de libertés politiques est bien reconnaissable. Le FSB (Service fédéral de sécurité) fait renverser par une voiture la fille d'un fonctionnaire qui voulait s'opposer à la corruption. Les miliciens en civil rossent les opposants politiques jusqu’à les laisser invalides. Un journaliste explose dans sa voiture piégée.

5. Sur le traitement du temps dans les romans de Chichkine, voir : Светлана Николаевна Лашова, «Принцип пазла. Язык и хронотоп в прозе М. Шишкина» («Le principe du puzzle. La langue et le chronotope dans la prose de M. Chichkine»), Вестник Пермского университета Le messager de l'université de Perm, $\mathrm{n}^{\circ}$ 6, vol. I2, 2010 (<http://www.rfp.psu.ru/archive/6.2010/ lashova.pdf $>$ ).

6. Chichkine travaille à partir de documents, tels que mémoires, journaux, témoignages, dont il réorganise la matière, de sorte que la frontière entre réalité et fiction est indiscernable. 
Les tensions ethniques ou mafieuses évoquées sont caractéristiques de la Russie postsoviétique : un homme menacé de mort par ses créditeurs se retrouve au cœur d'une rivalité mafieuse entre Toungouzes et Orotches (p. 107). Les Tchétchènes mettent à sac une maison, tuent la mère, avant de mettre le feu. La guerre russo-tchétchène, qui constitue l'une des lignes de force du roman, est reprise plus loin, lorsque le drogman se remémore l'épisode de sa vie où il fait visionner à un invité une cassette vidéo sur les atrocités de cette guerre. L'univers militaire est particulièrement prégnant, avec la confession d'un jeune vétéran de la guerre en Afghanistan, l'évocation des tortures qu'il a infligées aux prisonniers, le souvenir des civils tués par erreur (p. 52-53).

Cet univers sordide et immonde est peuplé d'ivrognes vautrés dans la rue, de misérables se déchirant dans des scènes de ménage, de jeunes qui font dérailler les trains. Les femmes sont prostituées, victimes de viols, contraintes aux avortements, elles manquent des soins les plus élémentaires, telle cette SDF ivre, qui accouche dans un wagon du métro en pleine nuit (p. I23), et elles n'échappent pas à la violence de l'univers carcéral. Les camps de femmes sont dépeints par petites touches précises, sans épargner aucun détail, mais le comble de l'humiliation est atteint dans les camps d'hommes, où les prisonniers eux-mêmes instaurent entre eux un ordre hiérarchique strict, de véritables castes, où les "coqs» sont les parias, les souffre-douleur des autres prisonniers (p. 69).

Les désordres de la dissolution de l'empire pourraient susciter chez le narrateur une forme de nostalgie de l'époque soviétique, mais il n'en est rien. Des bribes de la réalité soviétique affleurent non seulement dans les récits de vie des réfugiés, mais aussi dans les souvenirs personnels du drogman. Cette période apparaît comme tout aussi cruelle et immorale. Les enfants sont les premières victimes, comme en témoignent les souvenirs d'une réfugiée qui raconte son enfance dans un orphelinat, ou encore l'évocation du travail à l'usine, dont les vapeurs provoquent des intoxications. Il est question également des nombreux incendies criminels provoqués par envie ou jalousie (p. II2-II3), de la rafle des enfants juifs par les Allemands, et des persécutions contre les prêtres et leurs familles (p. 59).

Il n'y a donc pas de rupture entre la Russie contemporaine et l'URSS. La guerre russo-tchétchène actuelle n'est qu'un nouvel épisode d'une épopée plus ancienne. En effet, un des points culminants du roman est constitué par le récit de la déportation des Tchétchènes, ordonnée par les Soviétiques, et de l'extermination des civils récalcitrants et impotents dans un incendie volontaire, assorti d'une liste exhaustive des victimes, précisant leur âge : nourrissons, femmes, vieillards (p. 259-26I). 
Le regard sur la Russie soviétique de Bella, dans la seconde partie de son journal intime, pourrait être plus nostalgique. Revenue vivre en URSS, après un long séjour à Paris avec son mari, un haut fonctionnaire du nouveau régime, Bella mène la vie des privilégiés du nouveau régime. Sa description d'un jubilée, véritable "festin pendant la peste», à l'hôtel Métropole, le lieu le plus élitiste de l'Empire soviétique, donne l'image des excès de luxe d'une société décadente, à l'approche de la catastrophe :

Je les regardais s'empiffrer, siffler verre après verre, danser à perdre haleine. Comme si tout devait s'arrêter demain. Comme s'il fallait se dépêcher de profiter de tout aujourd'hui. Tous festoyaient et s'enivraient jusqu'à n'en plus pouvoir, jusqu'à perdre conscience, jusqu'à la nausée.

Iossif n’avait pas lésiné. Il avait choisi le Métropole, car les autres restaurants n'étaient pas à la hauteur. Partout il y avait des tapis, du cristal, le grand hall était illuminé, les portiers en uniformes galonnés. Les toilettes des dames n'étaient pas de la confection mais venaient de chez Lamanova. C'était partout un brouhaha de conversations, de rires, des effluves de parfum de luxe. Le champagne coulait à flot. Et au milieu il y avait la fameuse fontaine où étaient tombés tant de beaux messieurs et de belles dames. Et où il en tomberait encore beaucoup. (p. 386-387)

L'envers du décor est aussi apocalyptique, avec les corps gisant pêle-mêle :

Ce qui m’a fatiguée, ce sont surtout les déplacements, les trains, les wagons pleins de courants d'air et qui sentent le linge humide [...]. La correspondance en pleine nuit à Koursk était un cauchemar - les gens dormaient pêle-mêle sur le sol, serrant contre eux leurs baluchons, les toilettes étaient nauséabondes, l'atmosphère sinistre. [...] Devant les innombrables débits de boisson, étaient attroupés des hommes déguenillés et crasseux. On voyait plus d'ivrognes que de personnes sobres. (p. 389)

Les femmes soviétiques sont transformées en statues de pierre, comme après la malédiction qui a frappé Sodome et Gomorrhe :

Quand j'étais petite, papa nous a emmenées plusieurs fois voir des fouilles dans la steppe et nous a montré des idoles de pierre. Ces figures féminines que l'on trouvait dans les tumulus avaient pour nous quelque chose d'énigmatique, de mystérieux, d'éternel. Mais maintenant, j'en ai vu tellement de ces femmes de pierre. Dans toute la Russie, à chaque passage à niveau, elles regardent passer le train - des femmes ordinaires, en bottes de grosse toile, veste molletonnée et fichu gris, mais tout à fait semblables à ces figures de pierre. (p. 390)

C'est encore un décor de ruines, à l'image de la Russie soviétisée, que découvre Bella au cours d'une excursion dans un ancien manoir aristocratique :

Le parc est grand et beau, mais les statues sont renversées et brisées. L'étang en fer à cheval n' est plus qu'un marais stagnant. Les arbres sont creux et ne tiennent plus que par l'écorce. Les passerelles sont effondrées. Les habitants des environs ont pillé et saccagé tout ce qu'ils ont pu. J'essayais d'imaginer à quoi cela ressemblait avant. (p. 392) 
Ces ruines, ce sont celles de la Russie impériale, qui en I9I4, lorsque Bella commence son journal intime, était sur le point de s'effondrer. L'insouciance et la douceur de vivre, l'amour des parents (la nounou, l'église, les anges), laissent bientôt la place à l'inquiétude. Le voile de l'enfance se déchire, laissant percer, comme en écho des événements historiques en train de se produire, les mots "grève », «révolution", "pogrom» (p. Ioo). Bella accrédite la comparaison entre le déclin de l'empire tsariste et celui des empires de l'Antiquité : «Dans mon cerveau germe une idée lumineuse : peut être qu'un jour on considérera que nous-aussi étions des Grecs anciens qui vivaient parmi les barbares?» (p. 97) Au fil du texte, les souvenirs de Bella montrent sous un jour de plus en plus violent et cruel les bouleversements historiques - la guerre de I9I4, la prise du pouvoir par les bolcheviks, les exécutions, les luttes ethniques lors de la guerre civile :

Cela fait longtemps que les paysans russes guignent leurs terres, c'est pourquoi ils ont soutenu les bolchéviks et exterminé les Kalmouks. [...] Tout est souillé, barbouillé d'immondices. On voit des statues de Bouddha brisées. Des livres sacrés déchirés. [...] Mon dieu, les hommes sont devenus des bêtes féroces! (p. 267)

Il n'est pas anodin que Bella, alors qu'elle feuillette l'Évangile, s'arrête précisément sur la description de l'Apocalypse. Ce texte lui inspire la réflexion suivante :

C'est pourquoi le plus difficile à admettre dans l'Apocalypse, c'est qu'elle n'aura pas lieu [...] L'Apocalypse, en fait, nous la vivons ici, au quotidien, dans le gel et le blizzard, seulement elle est étalée dans le temps. Tout le monde meurt, mais pas au même moment. Mais finalement, cela revient au même, puisque de toute façon, ce sont des mondes entiers, des générations, des empires qui s'en vont ainsi. Où est Byzance? Où sont les Romains? Où sont les Hellènes? Pff, plus rien. Il ne reste rien ni personne, ni vainqueurs, ni vaincus. (p. 218)

En effet, le motif de l'Apocalypse est récurrent dans le roman. L'épigraphe est tirée du Livre de Baruch ${ }^{7}$, dans lequel Dieu lui-même révèle au prophète la succession des empires, jusqu'à la venue du Messie, qui signera la fin des temps.

Autre élément emblématique de la fin des temps, la ville de Pompéi figée par l'éruption du Vésuve apparait dans l'évocation, par une réfugiée, du tableau de Brioullov Le Dernier Jour de Pompéi.

On conduisait sa classe au musée et là-bas, sur le tableau Le Dernier Jour de Pompéi, les gens étaient sur le point de mourir, ils allaient être anéantis dans quelques

7. Il s'agit d'un livre deutérocanonique attribué à Baruch ben Neria, disciple de Jérémie, qu'il suivit en Égypte lors de la prise de Jérusalem par Nabuchodonosor. Il publie ses prophéties à Babylone, où il a finalement rejoint les Judéens captifs. Notons que le dernier vers "Car le Verbe a créé le monde et par le Verbe nous ressusciterons» ne figure pas dans la version que nous avons consultée en russe. Seuls les trois premiers vers sont attribués à Dieu qui s'adresse à Baruch (<http://znak-protest.hı6.ru/oo4/346.shtml>). 
instants. Mais l'année suivante, on les y emmenait à nouveau et sur le tableau, les derniers instants étaient toujours là. (p. II9)

Puis elle revient par deux fois dans les pensées du drogman :

Dans les fouilles de Pompéi on a trouvé des hommes creux ${ }^{8}$. (p. 378)

Le vide des hommes creux trouvés à Pompéi. (p. 42I-422)

De même que Pompéi est recouverte par les cendres du volcan, la Russie est (bien souvent) recouverte par la neige, motif essentiel du roman. La neige tombe en Suisse, exactement comme à Moscou. Le drogman la regarde tomber dans son studio en face du cimetière, et se remémore sa vie moscovite et son institutrice Galina Petrovna, dite Galpetra (p. 28). La neige recouvre tout. Elle permet l'oubli, elle cache les souffrances et les douleurs. Elle offre comme une page vierge sur laquelle pourra être écrite une nouvelle histoire. La neige peut prendre la forme de crayons blancs, que la vieille du septième étage laisse tomber du haut de sa fenêtre (p. 346). Sur la neige, comme sur une page blanche, on peut laisser une trace, la trace d'une vie. Parfois, le mouvement de la neige s'inverse, et elle tombe alors de bas en haut, et c'est comme feuilleter le livre en sens inverse (p. 347). La fonction de la neige est à la fois apocalyptique et rédemptrice.

Si la neige est le symbole de l'hiver, du temps arrêté et figé de l'empire millénariste, la fonte des neiges signifie le printemps et la résurgence de la vie; la fin de l'empire de l'hiver, sa débâcle. C'est la révolution (p. 36I-362), et c'est aussi le dégel.

Mais la pluie n'est pas toujours vivifiante, elle peut être la cause d'inondations aux dimensions bibliques, comme celle de Saint-Pétersbourg en 1924, dans les souvenirs de Bella : "C'est le déluge. Oui, c'est la fin du monde. Le châtiment de Dieu.» (p. 315)

La pluie est souvent associée à la souffrance et aux larmes, tant dans les souvenirs de Bella (p. 297, 303, 305, 306), que dans les récits des réfugiés, comme celui de cette femme qui porte le fardeau de la mort accidentelle de sa petite sœur, dont la tête a heurté violemment le sol, et du désespoir de sa mère :

Sur ces entrefaites, il s'était mis à pleuvoir et maman avait imaginé que si elle emmenait Sacha dehors, la pluie l'apaiserait. [...] Les branches ployaient, les arbres frémissaient, l'eau glougloutait dans les flaques et le bruit de la pluie avait tout de suite calmé Sacha. Il pleuvait fort et de biais, maman avait oublié de fermer les fenêtres et la terrasse était toute inondée. (p. 327)

Le drogman a connu maintes épreuves, et il pleure sur les ruines de son couple en regardant tomber la pluie dans le petit port italien de Massa

8. Ces «hommes creux» rappellent les «arbres creux» de l'extrait cité ci-dessus. 
Lubrense. Sous la pluie de l'Italie, il retrouve également le souvenir infiniment triste de son tout premier amour, pour une jeune fille qui s'est suicidée.

Bella elle aussi a connu un sort tragique. Pourtant le récit de sa vie laisse une impression de clarté et d'achèvement. C'est l'éducation sentimentale d'une jeune fille qui découvre l'amour, puis d'une femme qui s'épanouit dans la maternité et dans l'art, et enfin d'une vieille qui, malgré la lente dégradation de sa vie, la ruine de son corps, et sa mort, renaît dans ses souvenirs grâce à l'écriture. Cette promesse de renaissance dans la mort se trouve également inscrite en filigrane dans le récit d'un des réfugiés russes :

Moi, je le regardais et je me souvenais avoir vu à la télévision quautrefois on enterrait les morts assis, en position foetale, les jambes repliées contre la poitrine, comme pour que la personne puisse naître une deuxième fois. Si bien que la tombe était comme un utérus. C'est-à-dire que la mise au tombeau était comme un accouplement avec la terre, une sorte de fécondation de la terre par l'homme. (p. 66)

Comme la neige, la pluie est donc ambivalente. Elle est le symbole de la renaissance dans la mort, de la résurrection dans l'apocalypse, le symbole d'un temps des saisons, qui n'est pas linéaire, mais cyclique. Cette ambivalence est celle des sentiments du drogman à Rome, lorsqu'il s'y rend pour la première fois, avec sa femme Iseult, et que la pluie y est mêlée de soleil :

De la première Rome restait une impression de pluie mêlée de soleil. Le chemisier trempé d'Iseult plaqué contre sa peau et qu'elle essayait de décoller en tirant sur le tissu. Le bruit des pneus passant avec un chuintement particulier, une sorte de sifflement humide sur les pavés encore mouillés, mais brillant déjà au soleil. Sur les feuilles, les pierres, les murs trempés, ruisselait un soleil liquide et aveuglant. (p. 155)

\section{Rome, le temps arrêté et la remontée des temps}

Rome est centrale dans le roman de Chichkine. Le narrateur s'y rend à plusieurs reprises, en réalité et par la pensée. C'est de Rome qu'il écrit une lettre à son fils, "du haut d'un toit" (p. I52), alors qu’il s'est déjà séparé de sa femme Iseult, avec laquelle il était venu à Rome pour la première fois. Iseult, elle, était venue auparavant, avec son premier mari, Tristan, qui a péri dans une catastrophe automobile au cours de ce voyage (p. I60).

Tout particulièrement pour la littérature russe, Rome est la ville symbole de l'empire. Mais comme le fait remarquer Laure Troubetskoy, Chichkine se démarque de la tradition littéraire russe`. En effet, dans le roman de Chichkine,

9. L. Troubetzkoy, "Tela kamennye no telesnye. Obraz Rima v romane Šiškina» ("Corps de pierre mais de chair. L'image de Rome dans le roman de Chichkine»), Toronto Slavic Quartely, $\mathrm{n}^{\circ}$ 37, 20II, <http://www.utoronto.ca/tsq/2I/troubetzkoy2I.shtml>. 
Rome est, certes, la Rome des musées, en particulier ceux du Vatican, où les statues sont figées, immobiles, mais seulement jusqu'à ce que l'imagination et la parole leur redonnent une histoire et une vie :

Toutes les sculptures avaient été autrefois des dieux ou des hommes, à présent transformés en statues de sel qu'on avait rassemblées ici. Des cadavres de marbre, alignés comme une garde d'honneur à l'entrée du royaume des morts. Iseult avait imaginé de les ranimer en leur attribuant à chacun une histoire. [...] Ils avançaient ainsi en ressuscitant les morts. (p. 162-163)

Rome, la ville universelle, est aussi le lieu d'une quête impossible, celle du Vrai. Alors que le drogman croit avoir enfin trouvé, dans l'église SaintClément, les reliques de Cyrille, l'inventeur de l'alphabet cyrillique, Iseult lui apprend que ni ces reliques, ni le martyre de Clément, ni même la présence de saint Pierre à Rome ne sont attestés par les historiens (p. 170). La vérité ne cesse de se dérober, les statues ne sont que des copies d'originaux plus anciens et disparus : "Même le Tibre semblait une pâle copie d'un original disparu." (p. I64) Rome résonne de mélodies d'amour : «Pendant qu’ils traversaient la place, on entendait une musique italienne tonitruante venue d'on ne sait où, avec les mots amore, amore, amore qui revenaient tout le temps.» (p. 167) Or l'amour est aussi la quête impossible d'un original manquant. Celui du drogman pour Iseult lui apparaît comme une copie affadie de l'amour de Tristan et Iseult. "Il devait enlever Iseult à Tristan, l'arracher à leur Rome pour lui en faire connaître une autre.» (p. I68) «Et même le drogman n'était finalement que la copie d'un original perdu» (p. I65). «Il fallait que tu sois son Tristan. Il fallait que tu sois lui» (p. 415), «Parce que tu étais son Tristan, mais tu ne l'as pas compris", se reproche-t-il dans son soliloque final (p. 4I7).

Pour le drogman, Rome n'est pas très différente de Moscou, la ville de son enfance et de sa jeunesse : «Les odeurs et les bruits sont romains, mais la couleur des maisons est tout à fait moscovite.» (p. I53) Rome lui semble même par moments la copie de Moscou. Ainsi la statue romaine de l'Apollon du Belvédère est, pour le drogman, «la copie de celui qui était sous la neige à Ostankino». Les pigeons sont aussi "tout à fait comme ceux de Moscou» (p. 4I3). Comme Moscou, Rome a des "banlieues sales", et des "arrières cours mornes et sordides» (p. I6I). Dans la cohue de la rue romaine, le drogman aperçoit une vieille femme dont il «jurerait qu'elle sort du passage souterrain du métro Élektrozavodskaïa en ayant juste appris deux mots d'italien» (p. I54). En se promenant dans Rome, il se remémore les sorties de classe au musée Pouchkine, à Moscou, jusqu'à faire surgir par l'imagination son enseignante Galpetra. Ainsi, l'auteur du Cheveu de Vénus renouvelle le thème de Moscou - troisième Rome. Rome et Moscou sont semblables, non pas en tant que deux hypostases de l'empire, mais en tant que lieux de vie et d'amour pour le drogman. 
Loin d'être une ville-musée, Rome est grouillante et vivante. Elle est emplie d'odeurs ("odeurs d'essence, de café s'échappant par les portes grandes ouvertes des bars, d'encens et de cierges des églises, de parfum émanant des boutiques, d'urine et de chaux devant les porches», p. I53), de bruits («les cris des chats célébrant d'invisibles noces félines", p. 157), de taches colorées fugitives. C'est un amoncellement, un véritable chaos :

Rome des corps. Des corps partout - en pierre, mais charnels - corps d'hommes, de femmes, d'animaux à demi-humains. Muscles, seins, tétons, nombrils, fesses, - des dioscures, des empereurs, des tritons, des dieux, des faunes, des saints. Hanches, genoux, millets, talons, orteils écartés. (p. 156)

Cette description de la capitale de l'empire, boursouflée et bourgeonnante, pourrait être de la plume de Gogol, qui décrit de façon similaire, au début des Nouvelles de Saint-Pétersbourg, la perspective Nevski, avenue principale de l'autre capitale russe, celle des tsars ${ }^{10}$. L'ombre de Gogol ${ }^{\text {II }}$ accompagne le drogman dans ses promenades et dans ses réflexions. Dès les premières pages, au détour d'une relecture de procès-verbal d'interrogatoire, l'écrivain russe et son célèbre «Nez» se trouvent insérés dans le flux littéraire :

En classe on étudiait Gogol. Le jeune professeur expliquait que la fugue du nez était une tentative de fuir la mort et que sa réapparition signifiait le retour à l'ordre naturel de la vie et à la condition de mortel. (p. 37)

C'est à la recherche de la maison de l'écrivain que le drogman parcourt la via Sistina :

La via Sistina mène chez Gogol. [...] Si vous saviez avec quelle joie j'ai quitté la Suisse pour voler vers ma chère, ma magnifique Italie. Elle est à moi! Personne ne pourra me la prendre. Je suis né ici. La Russie, Pétersbourg, les neiges, les scélérats, l'enseignement, le thêâtre, tout cela n'était qu'un songe. (p. I54)

Le drogman, qui cite ici en les reprenant à son compte les mots d'une lettre de l'écrivain russe, semble s'identifier à lui.

Si la vie à Rome est dense au point d'éclater en «mille fragments» (p. 156), c'est que la cité éternelle est le seul endroit au monde, où le temps ne s'écoule pas, mais déborde.

Parce que s'il existe vraiment quelque chose d'authentique, on le cherche non pas là où on l'a perdu, mais à Rome, où le temps se comporte bizarrement - il

Io. Saint-Pétersbourg pourrait être cette «quatrième Rome» qui, selon Philotée, n’aurait pas dû advenir, d'où la malédiction qui la poursuit.

II. Gogol est aussi un émigré de l'empire. Oppressé par la vie à Saint-Pétersbourg, il avait trouvé refuge en Italie, à Rome, où il a passé plusieurs années de sa vie. Le style de Gogol, à la fois léger, humoristique, et tragique, avec ses "arabesques» et son caractère fantastique jouant sur la réalité et les illusions, est finalement assez proche de celui de Chichkine. Sur Gogol, voir l'ouvrage d'un autre émigré de l'empire (soviétique), Tertz Abram [Siniavski], Dans l'ombre de Gogol, trad. du russe par G. Nivat, Seuil, 1978. 
ne s'écoule pas, mais s'accumule, remplit la ville à ras bords, comme si on avait bouché la bonde avec le Colisée. (p. 428)

Les époques, au lieu de se succéder les unes aux autres, s'empilent l'une au dessus de l'autre, à la manière de la spirale de la colonne de Trajan, qui «se déroule sans fin vers le haut, symbole du mouvement, du progrès" (p. 158). À Rome, les époques, non seulement se superposent, mais s'imbriquent l'une dans l'autre, comme un kaléidoscope, si bien que tout près de la Rome chrétienne se trouve la Rome antique:

En contrebas, à huit ou dix mètres en dessous d'eux, s'étendait la Rome antique ou plutôt ses morceaux. Chaque ruine était éclairée. Les colonnes gisaient comme des os à moelle rongés. En attendant leur carre di agnello, ils buvaient du vin et lisaient tout haut le guide touristique à tour de rôle, essayant de comprendre comment était disposé tout ce qui était là deux mille ans avant eux, mais c'était impossible de s'y retrouver dans tous ces forums de Vespasien, d'Auguste, de César, de Nerva, imbriqués les uns dans les autres. (p. 157)

Toutefois, la Rome antique n'est pas plus authentique que la Rome de Cyrille et de saint Pierre. Loriginal est la Grèce antique : «Presque toutes les statues étaient des copies d'originaux grecs disparus.» (p. I63) Or, dans la tradition culturelle russe, la Grèce est d'abord le berceau de la foi orthodoxe byzantine, que la Russie a reçu en héritage de Constantinople, la «seconde Rome». Mais dans le roman, la réincarnation des Grecs, ce sont les Tchétchènes, ou encore les Orotches, c'est-à-dire les opprimés, les vaincus en fuite. En effet, l'Histoire se répète avec plusieurs variantes. Le roman ranime plusieurs couches de l'histoire entre la Russie et le Moyen-Orient, et on peut supposer que, confronté au grand nombre de réfugiés, russes ou tchétchènes, provenant du Caucase, le drogman est à la recherche d'une vérité concernant l'histoire de cette région.

Toutes ces histoires se ramènent à celle de la fuite de l'armée de mercenaires grecs levée par le roi perse Cyrus pour reprendre le pays à son frère Artaxerxès. Cette armée est menée par Xénophon, qui a relaté ce périple des Dix mille dans un récit intitulé l'Anabase. Or, le drogman, entre deux séances d'interrogatoire, s'adonne à la lecture de l'épopée de Xénophon, dont le texte $s^{\prime}$ intercale au sein des questions-réponses, jusqu'à les contaminer et se fondre avec elles ${ }^{12}$. Ainsi, le mot "anabase» - en grec ancien, la montée ou la remontée - utilisé par Xénophon pour désigner le retour en arrière de son armée défaite vers les détroits et la mer Noire, peut aussi désigner le procédé de retour en arrière dans le temps, dans la mémoire, la remontée de l'Histoire, pour trouver l'archétype de toutes les histoires. Des pages entières du roman

I2. Le texte de Xénophon envahit celui des interrogatoires : le récit de la mort de Cyrus au champ d'honneur prend la place d'une «question» (p. I2I). 
sont consacrées à l'épopée de Cyrus, une des nombreuses figures d'identification du drogman :

Avant d'éteindre la lumière et de mettre son oreiller sur son oreille, il a envie de se transporter à l'autre bout de l'empire et de parcourir avec Cyrus trente-cinq parasangs en cinq étapes dans le désert, avec l'Euphrate à main droite. (p. 27)

La figure de Cyrus, fondateur de l'empire perse, est emblématique du thème impérial. Mais en prenant Babylone en 540 avant J.-C., Cyrus avait mis fin à un autre empire, celui de Nabuchodonosor. Or, comme si le cours de sa vie était inversé par rapport à celui de l'Histoire, comme s'il devait remonter le temps, le drogman entretient une correspondance avec Nabuchodonosaure, - c'est ainsi qu'il appelle son fils (avec une déformation orthographique significative). Le nom de Nabuchodonosor est associé au mythe de Babylone, ville matrice du vice et de la corruption, symbole de la déchéance, de la chute. Mais Nabuchodonosor n'est pas le fossoyeur de Babylone, au contraire, c'est un faiseur d'empire, et son nom, titre de l'opéra patriotique de Verdi, Nabucco, qui évoque la naissance de l'Italie moderne, porte en lui à la fois le déclin d'un empire et la naissance d'un nouveau pays.

\section{L'écriture comme résurrection}

Pourtant, Nabuchodonosaure, le fils du drogman, n'est pas l'empereur puissant du Livre des Rois de l'Ancien testament. La terminaison de son nom évoque le dinosaure, véritable empereur du monde animal, dans un temps encore plus reculé. Il n'est qu'un enfant, qui règne sur un empire imaginaire :

Votre État nabuchodonosaurien, son glorieux passé géographique, le flux et le reflux de son histoire, les us de sa flore, les coutumes de sa faune, les volcans, les lois, les catapultes et les penchants anthropophages de sa population. Ainsi vous avez même des vampires et des draculas? (p. I5)

L'enfant règne sur un monde imaginaire, certes, mais compréhensible, organisé, stable. À l'inverse, «les empereurs grandissent vite et oublient leurs empires» (p. I5). La fin de l'empire peut être interprétée comme le passage à l'âge adulte. Grandir, c'est, comme pour Bella, abandonner ses illusions et la béatitude de l'enfance :

C'est étonnant comme je comprenais tout à l'époque, alors que maintenant je suis vieille, ma vie est derrière moi et je ne comprends plus rien. Comme si la vie était un passage de la compréhension à l'incompréhension. (p. 106)

La Suisse est un monde idéal comme celui de l'enfance, une île utopique, pour les réfugiés de l'ex-empire soviétique. Le drogman le dit dans sa lettre à 
son fils : «Bref cet empire est unanimement considéré par quelqu'un comme le meilleur des mondes.» (p. I6-I7) Toutefois, c'est avec ironie qu'il compare la Suisse au Paradis : "Alors c'est cela mon métier. Drogman du bureau des réfugiés du ministère de la Défense du paradis.» (p. i7)

"Ainsi vous affirmez que vous cherchez l'asile pour votre âme fourbue", demande le chef du service de la police des réfugiés, prénommé justement Pierre (p. 32). Or, saint Pierre ne laisse entrer personne. Il est froid et rigide, voire cruel avec les réfugiés, et va même jusqu'à les torturer par des questions impossibles, afin de démasquer leurs mensonges, trouver un prétexte pour leur refuser l'autorisation de séjour. Entre le Paradis et l'Enfer, entre la Suisse et la Russie, l'opposition n'est pas si évidente ${ }^{\mathrm{r} 3}$. Tout s'embrouille, le vrai et le faux, l'original et la copie, le bien et le mal, et il semble vain de tenter d'en démêler les fils.

Hanté par les histoires des réfugiés et par le quotidien sordide de son métier d'interprète, chargé du poids de tous les péchés et de tous les malheurs qu'il doit prendre sur lui en les écoutant et en les traduisant, le drogman se réfugie dans la lecture de l'Histoire idéale de Xénophon, qui pourrait bien mettre de l'ordre dans le monde. Mais l'Anabase de Xénophon resterait morte et figée comme les statues du Vatican, sans la réécriture du drogman, qui y insuffle les histoires des réfugiés, ainsi que sa propre histoire.

Comment raconter une histoire vraie, alors que la vie est insaisissable comme l'eau vive, qui coule et s'infiltre partout? Loin d'être réaliste, malgré la précision et l'abondance des traits qui cernent les contours de cette fin d'empire, le roman est une réflexion sur la littérature et ses rapports à la réalité et à la vie. L'image de l'hiver, qui court dans tout le roman, est facilement interprétée comme une métaphore de l'empire : "Vous débarquez comme martyrs de l'hiver", lance l'interrogateur à un demandeur d'asile, (p. 25). Mais l'hiver s'oppose au $m l y v o^{\mathrm{I4}}$ (p. IO8 et suiv.), c'est-à-dire au monde de l'imaginaire, de l'écriture, de la recréation artistique :

Tout disparaît dans l'hiver. L'été. L'enfance. [...] Mais dans l'hiver, quand le dégel est venu, tout ce zoo avec ses animaux, ses cages, ses odeurs, et cette caissière dans sa guérite, tout cela a fondu. Ils sont tous morts. - les animaux, les odeurs, la caissière. Tandis que dans le mlyvo, tout est resté intact, le zoo entier, et rien n'arrivera ni aux animaux, ni aux croûtes de pain détrempées dans l'eau noire, ni à la glace qui coule sur vos genoux, et la caissière continuera à enrager dans sa guérite et ne mourra jamais. (p. II7)

I3. Malgré l'assurance naïve de l'avocate commise d'office, qui vient rendre visite en prison à un réfugié débouté qui refuse de quitter le territoire suisse (p. 317-322).

I4. Ce mot désigne dans la mythologie des peuples de Sibérie, le royaume des morts. Loin d'être un paradis, un au-delà utopique, il coexiste avec le monde des vivants. 
L'hiver est donc la réalité, celle du temps qui passe et qui efface tout, où chacun est voué à la mort et à l'oubli. À l'inverse, dans le temps de l'imagination et de l'écriture ${ }^{\mathrm{I}}$, tout est éternel.

En racontant leurs histoires, même «s'ils ne sont pas ce qu'ils disent», les hommes sont vivants, car «leurs histoires elles, sont vraies» (p. 26). Le drogman rend la vie à Bella en étant le lecteur interprète de son journal, comme il jouerait une partition. La composition du roman peut d'ailleurs être comparée à une fugue musicale, caractérisée par des lignes mélodiques et des contrepoints, avec une alternance des thèmes épiques et lyriques, des tonalités majeure et mineure, des silences. Les motifs sont esquissés puis abandonnés, puis repris, jusqu'à se nouer, dans le soliloque final, en un véritable écheveau. On pourrait aussi comparer cette écriture au travail d'un archéologue, qui retire une à une les couches de poussière, remontant le temps, effectuant une "anabase», comme pour retrouver sous la neige de l'hiver les couleurs originelles. Cet «interprète» qui traduit la réalité en histoires, c'est l'écrivain qui, comme un chaman, a le pouvoir de passer d'un monde à un autre. Une des métaphores animales récurrentes est celle du chat qui regarde tomber les flocons de neige, en essayant de les attraper avec sa patte, assis tantôt sur le rebord intérieur de la fenêtre, tantôt sur le balcon (p. I26, 242, 335, 337). L'écrivain, comme ce chat, veut saisir une réalité insaisissable. C'est un embrouille-tout, dit Galpétra (qui, elle-même, perd la tête). "Tu mélanges tout! Tu as tout embrouillé! Tu es un embrouille-tout. L'embrouille-tout est au salon, il a mangé le melon.» (p. 4I4) Et plus loin :

Tu n'as rien compris du tout. [...] Les gens inventent Rome et ensuite ils s'étonnent de ne pas la trouver et de ne voir sur le Forum que des os rongés par le temps et envahis par l'herbe drue. (p. 4I5)

L'écrivain s'efforce de fabriquer le texte à partir des fils disparates des histoires $^{16}$. Patiemment, il s'applique à effacer les taches blanches sur la carte de son empire, à débusquer le vrai, l'authentique, cherchant à obtenir la «résurrection de la chair ${ }^{17} »$ :

I5. La métaphore de l'écriture, filée durant tout le roman, culmine dans le long soliloque final (p. 372-385) : "Le lendemain la plaine est recopiée au propre d'une ample écriture neigeuse. L'ombre d'un nuage certifie la neige conforme comme un tampon.» (p. 373) La neige et la mer se rejoignent ici en une "mer de neige", les isbas sont comparées à des "sous-marins des neiges» (p. 375).

I6. L'image des fils et des cheveux envahit littéralement le roman : fils brillants de la pluie, cheveux des femmes, etc.

17. "La résurrection de la chair» est le titre et le sujet biblique d'un tableau de Lucas Signorelli. Dans le roman, Tristan et Iseult étaient allés le contempler lorsque survint l'accident où Tristan trouva la mort et Iseult fut gravement blessée. Ce tableau est représenté sur la couverture du livre de Chichkine. 
La résurrection de la chair. De rien, du vide, d'un enduit blanc, d'un brouillard épais, d'un champ enneigé, d'une feuille de papier surgissent tout à coup des corps vivants qui se dressent et vont rester pour toujours, car c'est absolument impossible de disparaitre, de périr à nouveau, puisque la mort a déjà eu lieu. (p. 417-4I8)

À l'instar du temps romain, le temps du roman ne s'écoule pas, mais enfle jusqu'à déborder dans le soliloque final ${ }^{18}$. La machine s'emballe, les métaphores s'enchevêtrent, mais ne se tissent pas en un véritable texte, le sens des phrases est perdu, elles deviennent presque un balbutiement ${ }^{19}$. Cet apparent échec de la littérature signe peut-être le triomphe de la réalité et de la vie (et au fond, le triomphe du roman). Un certain mysticisme résonne dans l'hymne à l'herbe drue ${ }^{20}$ à la fin du roman. Le narrateur proclame que la mort n'existe pas : il faut croire en Dieu, c'est-à-dire en la vie, en l'herbe drue, gage d'éternité, qui repousse toujours sur les ruines et sur les corps morts.

Même le mlyvo, l'empire de la littérature, ne résiste pas à l'herbe drue, "qui pousse tranquillement et prend racine dans chaque fissure" (p. 424). La littérature permet la reconstruction d'un empire, une reprise de pouvoir de l'imagination sur la réalité (le Verbe permet la résurrection de la chair). Mais ce pouvoir est limité, la littérature ne peut pas véritablement ordonner le monde, répondre aux questions du bien et du mal, de la souffrance, de l'injustice, de la mort.

Le réalisme socialiste, qui, avec ses certitudes, était le visage littéraire de l'empire soviétique, est bien mort. Il a laissé la place au postmodernisme, forme d'apocalypse de la littérature, qui en proclame l'impuissance et la mort. Loin de s'y résigner, Chichkine a inventé un nouveau rapport de la littérature au temps (l'Histoire) et à la réalité (les histoires). En libérant l'écriture des contraintes spatio-temporelles du récit, il permet la résurrection de la langue littéraire ${ }^{21}$.

I8. Contrairement à Ulysse, de J. Joyce, qui présente pourtant aussi une écriture de type "courant de conscience", une alternance de modes de narration, une superposition de l'épopée et de la contemporanéité, une densité extrême de paraboles, allégories et symboles, mais qui se déroule sur un laps de temps précis d'une journée, le roman de Chichkine n'est pas divisé en chapitres et le temps de l'action est difficilement identifiable.

19. C'est plutôt un flot ininterrompu de paroles, exemple p. 425 : «On turbine pendant qu'ils dînent. Bien mal acquis ne profite jamais! À quoi bon se laver s'il n'y a personne à embrasser? Qui aime bien pressure bien. Le mandat d'expulsion est là, mais comment expulser des occupants invisibles?».

20. Le "Cheveu de Vénus", qui donne son titre au roman, est une herbe sauvage et résistante, symbole de la force de la nature.

2I. Sur le rôle et l'importance de la langue littéraire pour Chichkine, voir son interview : Шишкин Михаил, «“Язык - это оборона”. Михаил Шишкин о новом типе романа, русском языке и любви к Акакию Акакиевичу», Критическая масса, nº 2, 2005 . 